\title{
Endoscopic Treatment of Intrauterine Device Migration into the Bladder with Stone Formation
}

\author{
Masayuki Sano, MD, Kaoru Nemoto, MD, PhD, Takafumi Miura, MD, PhD, and Yasutomo Suzuki, MD, PhD
}

\begin{abstract}
Background: An intrauterine device is commonly used for contraception globally. Although intrauterine device placement is an effective and safe method of contraception, migration into the bladder with stone formation is a rare and serious complication. The management approaches for an intrauterine device embedded in the bladder include endoscopic procedures and open surgical removal. In this study, we report the case of a patient with recurrent urinary tract infection associated with intrauterine device migration and urolithiasis, who successfully underwent endoscopic treatment combined with laser fragmentation.

Case Presentation: A 22-year-old woman presented to our hospital with a 1-month history of lower abdominal pain, hematuria, and pain on urination. Transvaginal ultrasound showed a hyperechoic lesion in the bladder. A plain abdominal radiograph showed the presence of a T-shaped intrauterine device with calculus formation in the pelvis. CT revealed a vesical stone fixed to the top of the bladder wall, and there was no vesicovaginal fistula formation. She had undergone intrauterine device insertion several years previously. Cystoscopy confirmed the diagnosis. She underwent endoscopic lithotripsy, and the intrauterine device was extracted from the bladder wall. Repair of the bladder wall and disappearance of symptoms were confirmed.

Conclusion: Endoscopic treatment combined with laser fragmentation of stones surrounding a migrated intrauterine device should be considered as a minimally invasive approach, which can be performed safely.
\end{abstract}

Keywords: endoscopy, intrauterine device, migration, urolithiasis, laser lithotripsy

\section{Introduction and Background}

A N INTRAUTERINE DEVICE (IUD) is commonly used for contraception globally. Although an IUD is an effective and safe method of contraception, migration of the device into the bladder with stone formation is a rare and serious complication. ${ }^{1}$ The management approaches for an IUD embedded into the bladder are endoscopic procedures and open surgical removal. In this study, we report the case of a 22-year-old woman with recurrent urinary tract infection who successfully underwent endoscopic calculus fragmentation and IUD removal.

\section{Presentation of Case}

A 22-year-old Peruvian woman presented to the Department of Gynecology in our hospital with a 1-month history of lower abdominal pain, hematuria, and pain on urination. She came to Japan from Peru 2 years ago.

Pelvic examination results were normal, and urinary sediment revealed the presence of urine infection. The diagnosis was cystitis, and she was prescribed antibiotics. However, her symptoms did not improve. She was referred to a gynecologist again. Transvaginal ultrasound showed a hyperechoic lesion in the bladder. Therefore, she was referred to the Department of Urology.

Her laboratory data were normal. At urinalysis, many erythrocytes and leucocytes were found. A plain abdominal radiograph showed the presence of a T-shaped IUD with calculus formation in the pelvis (Fig. 1). CT revealed a vesical stone fixed to the top of the bladder wall, and there was no vesicovaginal fistula formation (Fig. 2). Cystoscopy confirmed the diagnosis of intravesical IUD migration complicated by a large bladder stone.

Department of Urology, Nippon Medical School Chiba Hokusoh Hospital, Chiba, Japan.

(C) Masayuki Sano et al. 2017; Published by Mary Ann Liebert, Inc. This is an Open Access article distributed under the terms of the Creative Commons Attribution License, which permits unrestricted use, distribution, and reproduction in any medium, provided the original work is properly cited. 


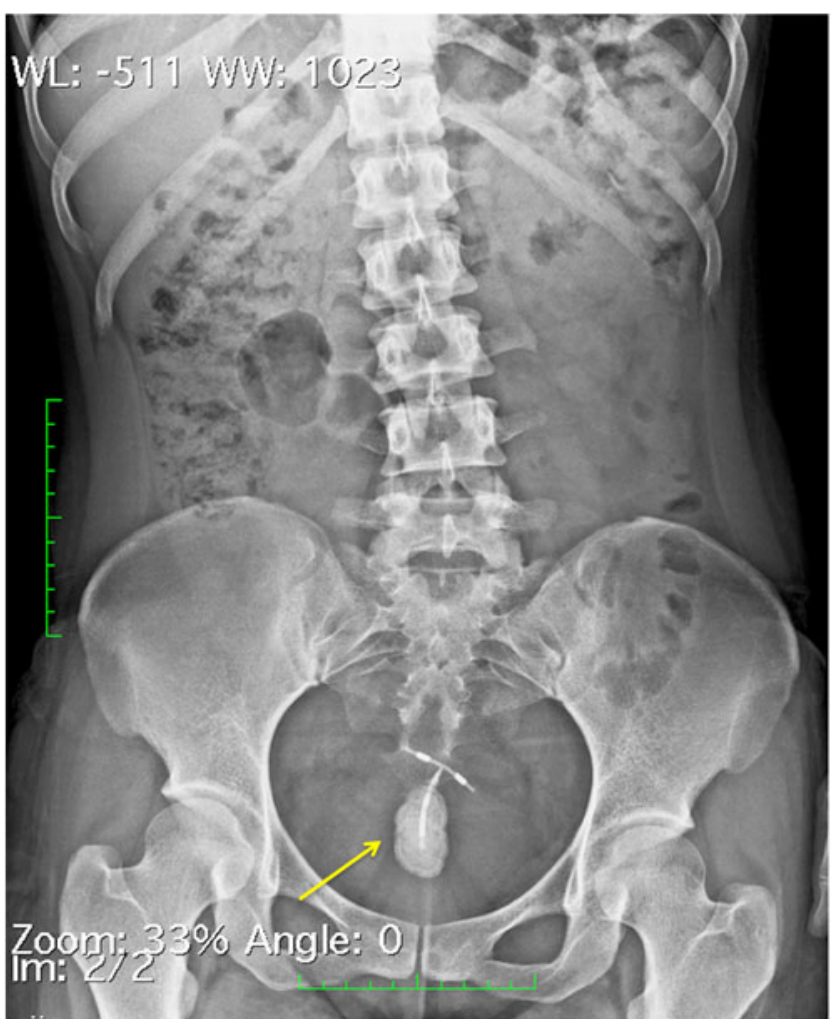

FIG. 1. Plain abdominal radiograph showing a T-shaped object with calculus formation in the pelvis (arrow).

On subsequent interview, she revealed that an IUD had been inserted in Peru. However, subsequently, she became pregnant and had an abortion. The IUD was not recognized at that point.

She underwent endoscopic laser lithotripsy (laser settings: Ho: $Y A G$ laser, energy $=2.0 \mathrm{~J} /$ pulse, rate $=10 \mathrm{~Hz}$, total energy $=$ $6.29 \mathrm{~kJ}$ ) under general anesthesia (Fig. 3A). The stone was completely fragmented, and then, the IUD was grasped with

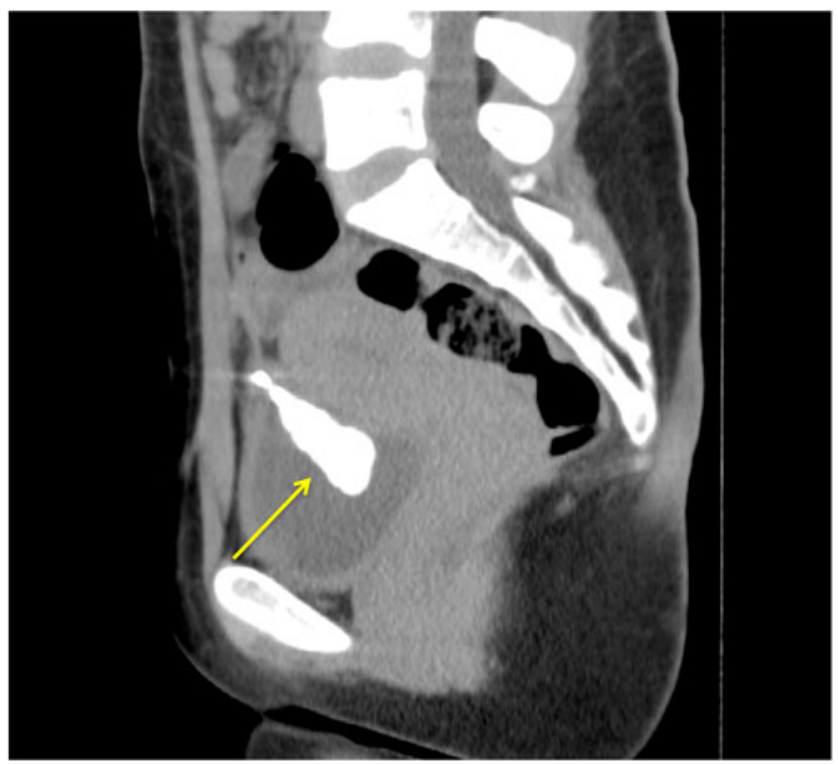

FIG. 2. A CT image of the intrauterine device that migrated into the bladder wall (arrow). forceps. With gentle traction, the IUD was extracted easily from the bladder wall and was removed (Fig. 3B).

A Foley catheter was left in the bladder for 2 days. During this period, hematuria was not observed. The patient was discharged on postoperative day 3 without any complication.

We confirmed repair of the bladder wall using cystoscopy and disappearance of symptoms at the outpatient clinic 1 month after IUD removal.

\section{Discussion and Literature Review}

IUD placement is a reversible contraceptive method widely used globally because of its low cost and complication rates. IUD migration into adjacent organs has been reported, including migration to the bladder wall with calculus formation.

Kassab et al. reported 165 cases of IUD migration. In this study, the IUD was located at the following sites: omentum, 45; rectosigmoid, 44; peritoneum, 41; bladder, 23; appendix, 8; small bowel, 2 ; adnexa, 1 ; and iliac vein, $1 .{ }^{2}$ IUD migration to the peritoneal cavity might cause adhesions, volvulus, fistula, and bowel perforation. ${ }^{3}$

Uterine perforation at the time of insertion, uterus/bladder contraction, gastrointestinal peristalsis, and peritoneal fluid movement are considered as possible mechanisms of IUD migration. ${ }^{4}$ Important triggers of perforation include insertion of the device by inexperienced persons, uterine size and position, inherent anatomic configuration, and resent abortion or pregnancy. ${ }^{3,4}$ The design and structural characteristics of the device as well as nature and rigidity or plasticity of the insert are considered to be the IUD-related factors. ${ }^{3}$

Because of intravesical migration, a patient can develop symptoms of urinary tract infection that are generally refractory to antibiotics. ${ }^{1}$ In the present case, the patient presented with repeated cystitis. If IUD migration to the bladder wall is confirmed, the migrated IUD should be removed as soon as possible because complications can occur. Extrauterine copper-laden devices may increase the possibility of inflammatory reactions. ${ }^{1}$

Transvaginal ultrasound, abdominal ultrasound, radiography, and pelvic CT are useful for determining the position of a migrated IUD in the bladder. In particular, CT is useful for diagnosing whether the IUD is penetrating surrounding organs.

Endoscopic surgery is generally selected for treatment, and open surgery is selected for cases involving large stones or partial penetration of the bladder wall. ${ }^{4}$ In recent years, laparoscopic surgery has supplanted open surgery owing to its minimally invasive approach. ${ }^{4}$ However, with the use of lithotripsy, IUD migration can be treated easily and appropriately, even if a large stone is present, as in our case.

\section{Conclusion}

In female patients with refractory pyuria and repeated cystitis symptoms, it is necessary to consider the possibility of IUD migration to the bladder. In addition, endoscopic treatment combined with laser fragmentation of stones surrounding the IUD should be considered as a minimally invasive approach, which can be performed safely.

\section{Disclosure Statement}

No competing financial interests exist. 

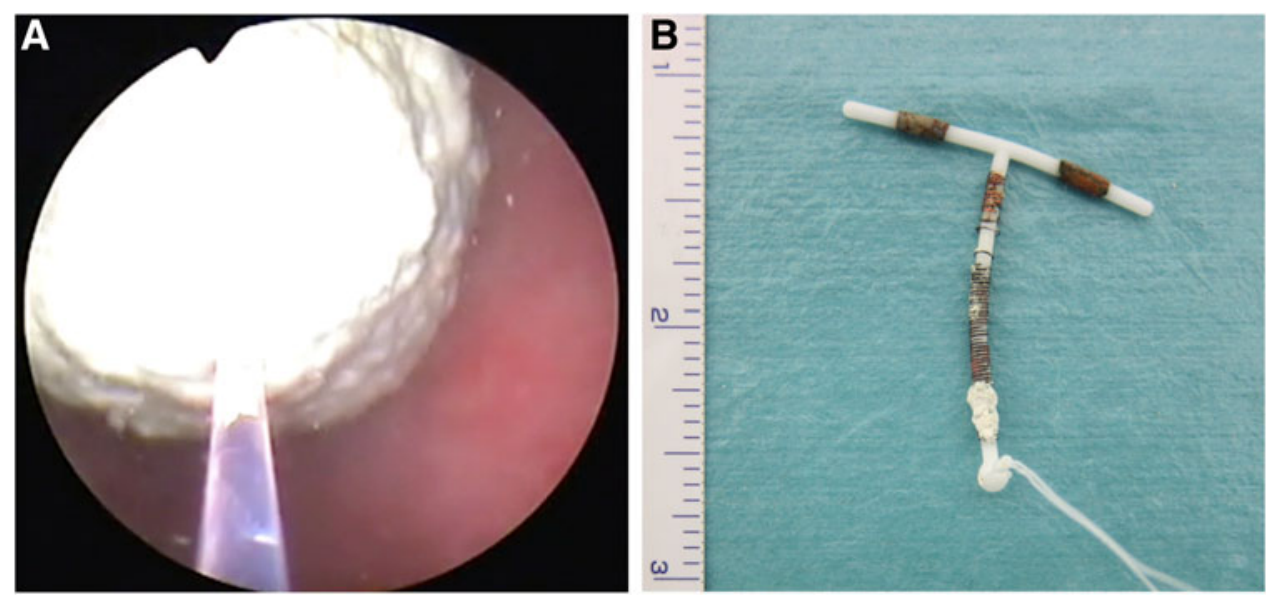

FIG. 3. (A) An image showing a bladder stone surrounding the intrauterine device; (B) an image showing the removed intrauterine device.

\section{References}

1. El-Diasty TA, Shokeir AA, El-Gharib MS, et al. Bladder stone: A complication of intravesical migration of lippes loop. Case report. Scand J urol Nephrol 1993;27:279-280.

2. Kassab B, Audra P. The migrating intrauterine device. Case report and review of the literature. Contracept Fertil Sex 1999; 10:696-700.

3. Inceboz US, Ozcakir HT, Uyar Y, et al. Migration of an intrauterine contraceptive device to the sigmoid colon: A case report. Eur J Contracept Reprod Health Care 2003;8: 229-232.

4. Shin DG, Kim TN, Lee W. Intrauterine device embedded into the bladder wall with stone formation: Laparoscopic removal is a minimally invasive alternative to open surgery. Int Urogynecol J 2012;23:1129-1131.

Address correspondence to: Masayuki Sano, MD Department of Urology Nippon Medical School Chiba Hokusoh Hospital 1715 Kamagari Inzai

Chiba 270-1694 Japan

E-mail: sano919@nms.ac.jp

Cite this article as: Sano M, Nemoto K, Miura T, Suzuki Y (2017) Endoscopic treatment of intrauterine device migration into the bladder with stone formation, Journal of Endourology Case Reports 3:1, 105-107, DOI: 10.1089/cren.2017.0038.

\section{Abbreviations Used \\ $\mathrm{CT}=$ computed tomography \\ IUD $=$ intrauterine device}

